\title{
Glucose 6-phosphate transport regulatory protein UhpA regulates the virulent genes in Edwardsiella piscicida
}

\section{Jiakang Chen}

Shandong Agricultural University

\section{Yongcan Sun}

Shandong Agricultural University

\section{Tingqi Ye}

Shandong Agricultural University

Xuepeng Wang ( $\nabla$ xpwang@sdau.edu.cn )

Shandong Agricultural University https://orcid.org/0000-0003-1189-8955

\section{Research}

Keywords: Edwardsiella piscicida, UhpA, virulent gene, Pathogenicity

Posted Date: April 14th, 2020

DOI: https://doi.org/10.21203/rs.3.rs-22255/v1

License: @ (i) This work is licensed under a Creative Commons Attribution 4.0 International License. Read Full License 


\section{Abstract}

Edwardsiella piscicida (E. piscicida) is an important zoonotic pathogen, which infects animals by colonizing the intestine. Glucose 6-phosphate (Glu6P) was an important carbohydrate in intestine and could be used as a regulate signal. Here we identify a virulence-regulating pathway named Glu6P transport regulatory protein UhpA, which affects the virulent genes of hemolysins, flagellar, T3SS, T6SS and metabolism related genes how to promote E. piscicida infect the host. The results showed that the metabolism related gene expression of cysteine synthase (orf 1134) and sulfate transporter (ychM) in the uhpA mutant strain $\triangle$ uhpA was 0.76 -fold and 0.68 -fold lower than the ones in the wild strains $(\mathrm{P}<0.05)$, the gene expression of eth $A$ and eth $B$ in the $\triangle u h p A$ strain was 0.80 -fold and 0.72 -fold lower than the ones in the wild strains $(\mathrm{P}<0.05)$. However, the gene expression of fliC and flgNin the $\Delta u h p A$ was 1.51 -fold and 1.21-fold higher than the ones in the wild strains $(\mathrm{P}<0.05)$, the gene expression of T3SS (esrB and esrC) and T6SS (evpB and evpC) in the $\triangle u h p A$ was 1.27-fold, 1.13-fold 1.28-fold and 1.23-fold higher than the ones in the wild strains $(P<0.05)$. Besides, the survival rate of fish challenged with E. piscicida EIB202 and $\triangle u h p A$ was $50 \%$ and $30 \%$ respectively. These suggested that although the uhpA gene deletion decreased the metabolic level and the hemolysins related gene expression in E. piscicida, the uhpA gene could down regulate the key virulent gene expression to decrease the pathogenicity of E. piscicida in fish.

\section{Importance}

Edwardsiella piscicida (E. piscicida) is an important zoonotic pathogen, which is widely distributed in aquatic environments and infects hosts mostly via the intestine. The intestine provides a nutritional and competitive environment for the pathogens. Successful colonization by pathogens requires scavenging nutrients, sensing chemical signals, competing with the resident bacteria and precisely regulating the expression of virulence genes. The Glucose-6-phosphate (Glu6P) in the intestine could trigger a novel signaling system named UhpA, which enables the cell to regulate the virulent genes. Here we found that the UhpA could down regulate the key virulent gene expression to decrease the pathogenicity of $E$. piscicida in fish.

\section{Introduction}

Edwardsiella piscicida (E. piscicida) is a rod-shaped Gram-negative, facultatively anaerobic, noncapsulated, motile, intracellular bacteria, which belong to an Enterobacteriaceae, Edwardsiella species, the old name was called as $E$. tarda $(1,2)$. This bacterium could infect fish and move through the food chain to infect amphibians, reptiles, and birds, and humans, which particularly infects fish and causes large economic losses $(3,4)$. E. piscicida infected host meanly through the intestine because it provides nutrition for bacteria $(5,6)$. The microbiotas rely on intestine nutrient availability and invading pathogens compete for these resources and regulate its virulent gene expression to enhance its pathogenicity (6-8). 
The pathogenesis of E. piscicida include many virulence factors such as the production of hemolysis ( 9 , 10), filament structural protein of flagellar (11), possession of type W secretion system (T3SS) $(2,9)$, and type VI secretion system (T6SS) (12), production of growth metabolism $(13,14)$, ability to adhere, invade, survive and replicate in host cells (2). Additionally, most of them were regulated by the two-component signal transduction system (TCS), such as UhpA/UhpB-UhpC, which enables the cell to acquire phosphorylated sugars from its environment that can be used as carbon or energy sources (15). Although uhpA gene deletion affects Glucose 6-phosphate (Glu6P) usage in E. piscicida and UhpA could decrease its pathogenicity in zebrafish (15), UhpA how to regulate the main virulent genes of hemolysis, flagellar, T3SS, T6SS and metabolism are still not available.

The study aims to find out the uhpA gene in E. piscicida on how to affect the main virulent gene expression and pathogenicity of E. piscicida. Elucidate the key virulent gene expression of hemolysis, flagellar, T3SS, T6SS and metabolism in the uhpA gene mutant strain and the wild type strain of $E$. piscicida. We believe that understanding this mechanism of E. piscicida would be helpful to explain the key role of UhpA in the pathogenicity of E. piscicida.

\section{Results}

\subsection{The uhpA gene deletion down-regulates the gene expression of metabolism}

To evaluate the function of the uhpA gene in E. piscicida how to affect the metabolism related genes. The gene expression was carried out in the uhpA mutant strain and the wildtype strain, and the results were shown in (Fig. 1). Results demonstrated that the metabolism related gene expression of cysteine synthase (orf 1134) and sulfate transporter ( $y$ chM) in the mutant strains was 0.76 -fold and 0.68 -fold lower than the ones in the wild strains $(P<0.05)$. This suggested that the uhpA gene deletion decreased the metabolic level E. piscicida.

\subsection{The uhpA gene deletion down-regulates the gene expression of hemolysis}

Hemolysins are lipids and proteins that cause lysis of red blood cells by destroying their cell membrane. The primary function of hemolysins is that of hemolysis, the result of two key hemolysis related gene expressions was shown in (Fig. 2). Results showed that the gene expression of ethA and eth B in the mutant strains was 0.80 -fold and 0.72 -fold lower than the ones in the wild strains $(P<0.05)$. This suggested that the uhpA gene deletion decreased the hemolysis related gene expression in E. piscicida.

\subsection{The uhpA gene deletion up-regulates the gene expression of flagellar}

The primary function of a flagellum is that of locomotion, the result of two flagellar related gene expressions was shown in (Fig. 3). Results showed that the gene expression of $f$ liC and $f l g N$ in the mutant strains was 1.51-fold and 1.21-fold higher than the ones in the wild strains $(P<0.05)$. This suggested that the uhpA gene deletion increased the flagellar related gene expression in E. piscicida.

\subsection{The uhpA gene deletion up-regulates the gene expression of T3SS and T6SS}


The result of T3SS and T6SS related gene expressions was shown in (Figs. 4 and 5). Results showed that the gene expression of T3SS (esrB and esrC) and T6SS (evpB and evpC) in the mutant strains was 1.27fold, 1.13-fold 1.28-fold and 1.23-fold higher than the ones in the wild strains $(P<0.05)$. This suggested that the uhpA gene deletion increased the T3SS and T6SS related gene expression in E. piscicida.

\subsection{The uhpA gene deletion of $E$. piscicida attenuates virulence in fish}

E. piscicida EIB202 caused mortalities in tilapia at first day after being orally injected infection, whereas the death occurred at 1-3 days after infection with the mutant strain E. piscicida $\triangle u h p A$ (Fig. 6). The survival rate of E. piscicida EIB202 and $\triangle$ uhpA groups was $50 \%$ and $30 \%$ respectively. None of the fish in the control group died and pure cultures of E. piscicida strains were recovered from the kidney and liver of dead fish.

\section{Discussion}

Host-bacterial interactions are very complex, the fundamental relationship between them are competition between host immunity and bacterial pathogenicity $(6,16-18)$. For example, host immunity after vaccination was greater than the invading ability of bacteria; fish could not be dead appearing $(4,19,20)$. As we know E. piscicida is an important zoonotic pathogen, although Edwardsialla has been known as a serious pathogen of aquatic animals for a long time, its pathogenicity mechanisms are yet to be fully elucidated. The pathogenesis of E. piscicida appears to be multifactorial virulence factors. One of them, the UhpA was regulated by the transport protein UhpT, to transport a broad range of phosphorylated sugars (15). In this system, the Glu6P has been used as a broad specificity of sugar phosphates (21), which decreased the pathogenicity of E. piscicida in zebrafish (15). Although UhpA decreased its pathogenicity has been reported, UhpA how to regulate the main virulent gene are still not available.

Cysteine synthase coded by open reading frame 1134 in E. piscicida genome, have been reported to participate in the regulation of ethanol utilization and production of antioxidant respectively (13). With the growing knowledge on the physiology of dissimilatory sulfate, one of them coded by $y c h \mathrm{M}$ gene has been identified in bacteria including E. piscicida $(14,22)$. The virulence element hemolysis (Eth) system, which comprised EthA and EthB, widely distributed in this bacterium, which is essential for the fish invasion in vivo and in vitro $(9,10)$. In this study, our results showed that the metabolism related gene and the hemolysis related gene expression were lower than the ones in the wild strains. This suggested that the uhpA gene deletion decreased the metabolic level and hemolysis ability in E. piscicida.

On the other hand, a flagellar protein encoded by flgN and fliC plays an important role in the adhesion and the proptosis in E. piscicida respectively $(11,23,24)$. Meanwhile, EsrA and EsrB are responsible for T3SS regulation in E. piscicida $(2,9)$. Also, EvpB and EvpC proteins regulated iron-restricted conditions in vitro had a crucial role in the T6SS mediated pathogenesis of E. piscicida (12). The result in this study showed that the flagellar related gene, T3SS and T6SS related gene expressions were higher than the ones in the wild strains. Importantly, the survival rate of fish infected with E. piscicida EIB202 was higher than fish infected with $\triangle$ uhpA. In general, the uhpA gene deletion more effect on the flagellar protein, T3SS and 
T6SS, enhanced the pathogenicity of E. piscicida. In summary, the pathogenicity of the E. piscicida mutant strain $\triangle u h p A$ increased because the key virulent gene upregulated after the uhpA gene deletion.

\section{Materials And Methods}

\subsection{Bacterial strains and culture conditions}

Bacterial strains include the wild type strain E. piscicida EIB202, the uhpA gene mutant strain $\triangle u h p A(15$, 25). E. piscicida strains were grown in tryptic soy broth (TSB) at $28^{\circ} \mathrm{C}$.

\subsection{The Expression Of Main Virulent Genes}

The E. piscicida EIB202 and $\triangle u h p A$ were grown in TSB cultures at $28^{\circ} \mathrm{C}$. Total RNA was extracted at 0.5 $\mathrm{OD}_{600}$ using a total RNA isolation system (TaKaRa company, Dalian, China). To verify that the RNA was free of DNA contamination, PCR amplification was performed using the RNA as a template. The primers for virulent genes are shown in (Table 1). The RT-PCR and qPCR was carried out performing our previous research $(8,26,27)$. 
Table 1

Primers used in this study

\begin{tabular}{|ll|}
\hline Primer names & Primer sequence (5'-3') \\
\hline EsrB-F & CGCCTACGTCCTGAAGCAA \\
\hline EsrB-R & CCTGAGCCATATCGGACGAG \\
\hline EsrC-R & AGGCTGGTCTTGGCCTACAG \\
\hline FliC-F & CGGTAGAGCGTGTCGAACAG \\
\hline FliC-R & ACCGCTTCACCGCCAATATCAAC \\
\hline FlgN-F & GATAGAGCCGTTCTGTGCCTGTAC \\
\hline FlgN-R & CCGTTGTGCTGATTCATGTCATGC \\
\hline EthA-F & CGGCTGCGGCTTTATCAATA \\
\hline EthA-R & ACCAGATCCAGCACGCTGTT \\
\hline EthB-F & CAGCCGCGACGTCAA \\
\hline EthB-R & CCCTCGATACGCTCGATAAA \\
\hline EvpB-F & AGCCGAGCCGATCTGATCCG \\
\hline EvpB-R & CCGCCGCAACGTGTGAGATC \\
\hline EvpC-F & GTCGCCGTCTCCAGCTATCAATG \\
\hline EvpC-R & CGCCTTGCCGTTCTGATCCTG \\
\hline 1134-F & CCGAGATCCACCGCCAGACC \\
\hline 1134-R & CTCGACGGCCACAATGCTGAC \\
\hline YchM-F & CGTCACCGATATGCCGTTCCAG \\
\hline YchM-R & TAGCGTTCCTCCTCCAGACTGC \\
\hline 16 s rRNA-F & ACCCTGGTAGTCCACGCTGTAAACG \\
\hline 16 s rRNA-R & CATCGAATTAAACCACATGCTCCACC \\
\hline
\end{tabular}

\subsection{Test Of Bacterial Virulence In Fish Model}

The tilapia was prepared as our previous paper and has been approved by the Animal Ethics Committee of Shandong Agricultural University (8), the mean weight of the tilapia was $73.09 \pm 3.07 \mathrm{~g}$, and the cultured water's temperature was $28.0 \pm 1.0^{\circ} \mathrm{C}$. These fish were recognized as disease-free animals to assess the virulence of E. piscicida strains (EIB202 and $\Delta u h p A$ ). Fish from each group were orally 
infected with $100 \mu \mathrm{L}$ PBS containing bacterial cells at $10^{10} \mathrm{CFU} / \mathrm{mL}$ according to the previous research. The number of dead fish was calculated observed over for 14 days.

\subsection{Statistical Analysis}

Statistical significance was determined by ANOVA analysis. Differences were considered significant at P $<0.05$.

\section{Declarations}

\section{Conflict of interest}

The authors declare that they have no conflict of interest.

\section{Availability of data and material}

All data generated or analyzed during this study are included in this published article.

\section{Acknowledgments}

This work was supported by the National Natural Science Foundation of China (No. 31402325), Natural Science Foundation of Shandong Province (ZR2019MC031), the earmarked fund for the Modern Agroindustry Technology Research System in Shandong Province (No. SDAIT-14-07), National Key R\&D Program of China冈2019YFD0900101) and funds of the Shandong "Double Tops" Program.

\section{References}

1. Abayneh T, Duncan JC, Henning S. Edwardsiella piscicida sp. nov., a novel species pathogenic to fish. J Appl Microbiol. 2013;114:644-54.

2. Leung K, Wang Q, Yang Z, Siame B. Edwardsiella piscicida: A versatile emerging pathogen of fish. Virulence. 2019;10(1):555-67.

3. Wang QY, Yang MJ, Xiao JF, Wu HZ, Wang X, Lv YZ, Xu LL, Zheng HJ, Wang SY, Zhao GP, Liu Q, Zhang YX. Genome Sequence of the Versatile Fish Pathogen Edwardsiella tarda Provides Insights into its Adaptation to Broad Host Ranges and Intracellular Niches. PLoS One. 2009;4:10-7646.

4. Yan MC, Liu JY, Li Y, Wang XP, Jiang H, Fang H, Guo ZM, Sun YC. Different concentrations of Edwardsiella tarda ghost vaccine induces immune responses in vivo and protects Sparus macrocephalus against a homologous challenge. Fish Shellfish Immunol. 2018;80:467-72.

5. Wang X, Lu R, Ding L, Yan M, Chai X, Chen S, Xie Q. 2012. Polysaccharides, Saponins and Water decoction of Astragalus membranaceus significantly enhance the non-specific immune response in Spotted maigre (Nibea albifiora). Israeli Journal of Aquaculture - Bamidgeh 64.

6. Pacheco AR, Curtis MM, Ritchie JM, Munera D, Waldor MK, Moreira CG, Sperandio V. Fucose sensing regulates bacterial intestinal colonization. Nature. 2012;492:113-7. 
7. Fischbach MA, Sonnenburg JL. Eating for two: how metabolism establishes interspecies interactions in the gut. Cell Host Microbe. 2011;10:336-47.

8. Wu J, Liu G, Sun Y, Wang X, Fang H, Jiang H, Guo Z, Dong J. The role of regulator FucP in Edwardsiella tarda pathogenesis and the inflammatory cytokine response in tilapia. Fish Shellfish Immunol. 2018;80:624-30.

9. Wang X, Wang QY, Xiao JF, Liu Q, Wu HZ. Hemolysin EthA in Edwardsiella tarda is essential for fish invasion in vivo and in vitro and regulated by two-component system EsrA-EsrB and nucleoid protein HhaEt. Fish Shellfish Immunol. 2010;29:1082-91.

10. Buján N, Toranzo AE, Magariños B. 2018. Edwardsiella piscicida: a significant bacterial pathogen of cultured fish. DISEASES OF AQUATIC ORGANISMS (Dis Aquat Org) 131:59-71.

11. He Y, Xu T, Fossheim LE, Zhang XH. 2012. FliC, a Flagellin Protein, Is Essential for the Growth and Virulence of Fish Pathogen Edwardsiella tarda. PLoS One 7.

12. Cui SI, Xiao JF, Wang QY, Zhang YX. H-NS binding to evpB and evpC and repressing T6SS expression in fish pathogen Edwardsiella piscicida. Arch Microbiol. 2016;198:653-61.

13. Hu YH, Li YX, Sun L. Edwardsiella tarda Hfq: impact on host infection and global protein expression. Vet Res. 2014;45:23.

14. Marietou A, Røy H, Jørgensen BB, Kjeldsen KU. Sulfate Transporters in Dissimilatory Sulfate Reducing Microorganisms: A Comparative Genomics Analysis. Frontiers in Microbioogyl. 2018;9:309.

15. Liu JY, Sun YC, Huang JJ, Ding L, Ye TQ, Wang XP. 2020. UhpA in Edwardsiella piscicida decreases the pathogenicity and the capability ofinducing cytokine response in zebrafish. Aquaculture Reports 100-293.

16. Wang X, Yan M, Hu W, Chen S, Zhang S, Xie Q. Visualization of Sparus macrocephalus Infection by GFP-Labeled Edwardsiella tarda. The Israeli Journal of Aquaculture-Bamidgeh. 2012;64:4038-47.

17. Pan $T$, Yan $M$, Chen $S$, Wang $X$. Effects of ten traditional Chinese herbs on immune response and disease resistance of Sciaenops ocellatus. Acta Ichthyologica ET Piscatoria. 2013;43:41-9.

18. Wang Q, Fu T, Li X, Luo Q, Huang J, Sun Y, Wang X. Cross-immunity in Nile tilapia vaccinated with Streptococcus agalactiae and Streptococcus iniae vaccines. Fish Shellfish Immunol. 2020;97:382-9.

19. Wang Q, Wang X, Wang X, Feng R, Luo Q, Huang J. Generation of a novel Streptococcus agalactiae ghost vaccine and examination of its immunogenicity against virulent challenge in tilapia. Fish Shellfish Immunol. 2018;81:49-56.

20. Wang Y, Wang X, Zhang B, Li Z, Yang L, Li X, Ma H. 2020. A lysin motif-containing protein (SpLysMD3) functions as a PRR involved in the antibacterial responses of mud crab, Scylla paramamosain. Fish \& Shellfish Immunology 97:257-67.

21. Västermark $\AA$, Saierr MHJ. The Involvement of Transport Proteins in Transcriptional and Metabolic Regulation. Curr Opin Microbiol. 2014;18:8-15.

22. Wang X, Lu C. Mice orally vaccinated with Edwardsiella tarda ghosts are significantly protected against infection. Vaccine. 2009;27:1571-8. 
23. Jiao XD, Hu YH, Sun L. 2010. Dissection and localization of the immunostimulating domain of Edwardsiella tarda FliC.Vaccine 28(34):5635-40.

24. Jiang X, Qin YX, Lin GF, Huang L, Huang B, Huang WS, Yan QP. FlgN plays important roles in the adhesion of Aeromonas hydrophila to host mucus. Genet Mol Res. 2015;14(2):6376-86.

25. Xiao JF, Wang QY, Liu Q, Wang X, Liu H, Zhang YX. Isolation and identifieation of fish pathogen Edwardsiella tarda from mariculture in China. AquaRes. 2008;40:13-7.

26. Chen J, Wang W, Wang X, Zhang Q, Ren Y, Song J, Wang X, Dong X, Huang J. First detection of yellow head virus genotype 3 (YHV-3) in cultured Penaeus monodon, mainland China. J Fish Dis. 2018;41(9):1449-51.

27. Yu J, Ji X, Wang X, Li T, Wang H, Zeng Q. Identification and characterization of differentially expressed genes in hepatopancreas of oriental river prawn Macrobrachium nipponense under nitrite stress. Fish Shellfish Immunol. 2019;87:144-54.

\section{Figures}

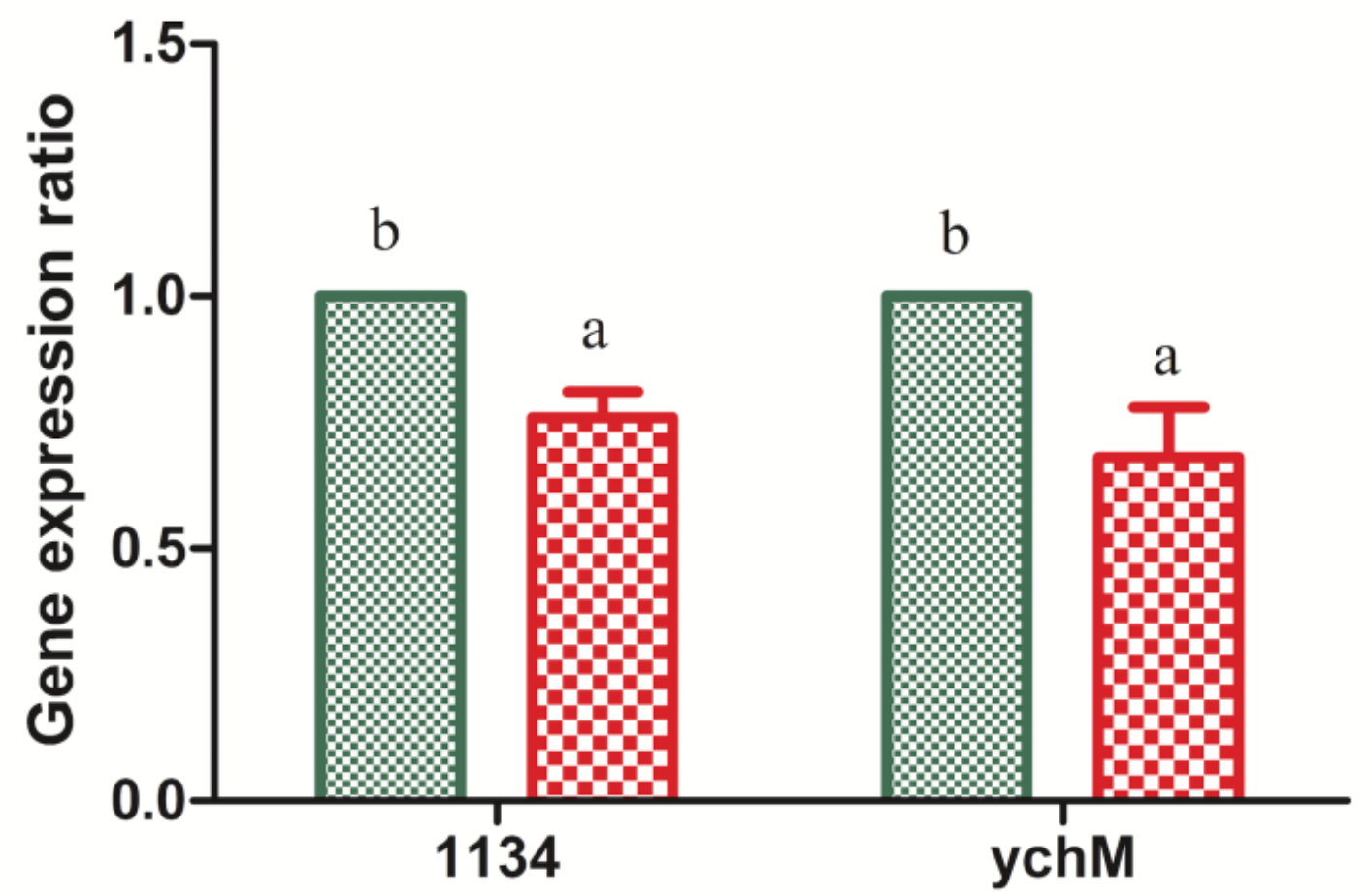

Figure 1

The difference of transcript levels of the cysteine synthase and sulfur metabolism related genes in $\mathrm{E}$. piscicida EIB202 and mutant strain $\triangle$ uhpA. Alphabet a and b: Values indicate significant difference at $\mathrm{P}<0.05$. 


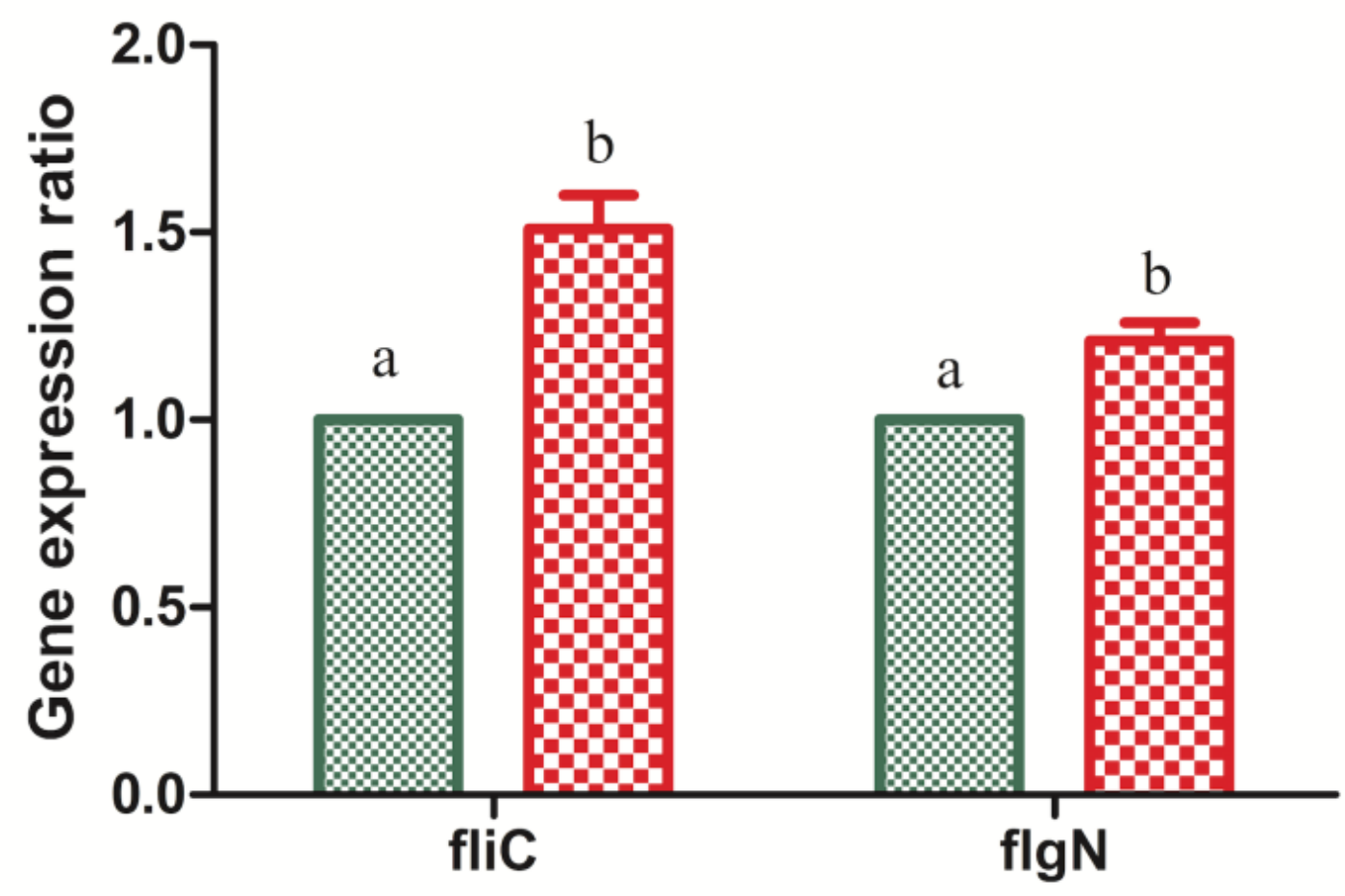

Figure 2

The difference of transcript levels of the flagellar related genes in E. piscicida EIB202 and mutant strain $\triangle$ uhpA. Alphabet a and b: Values indicate significant difference at $\mathrm{P}<0.05$.

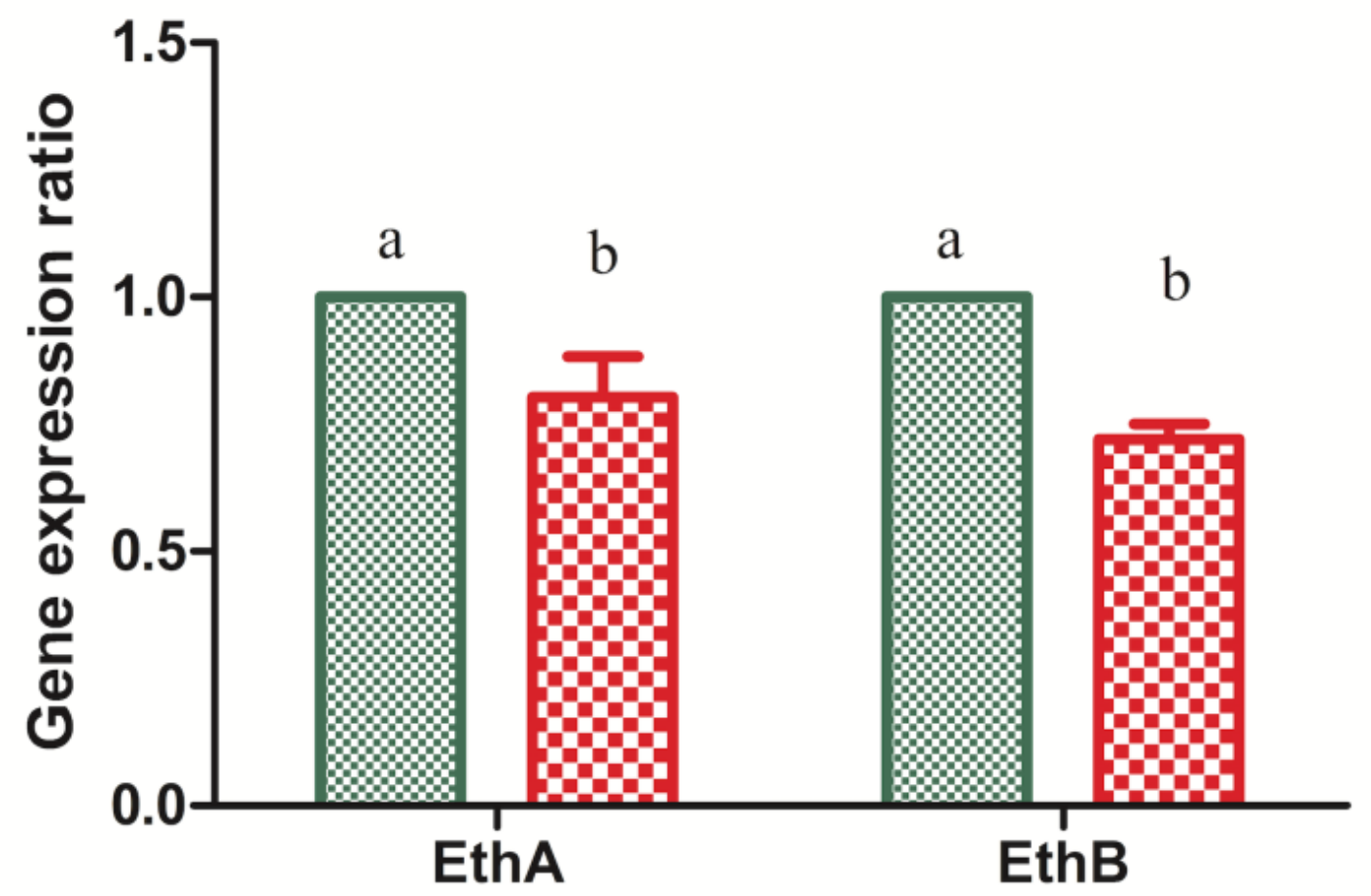

$\otimes \mathrm{EIB}_{202}{ }^{\mathrm{a}}$
$\triangle \mathrm{uhpA}$

Figure 3 
The difference of transcript levels of the hemolysis related genes in E. piscicida EIB202 and mutant strain $\triangle$ uhpA. Alphabet a and $\mathrm{b}$ : Values indicate significant difference at $\mathrm{P}<0.05$.

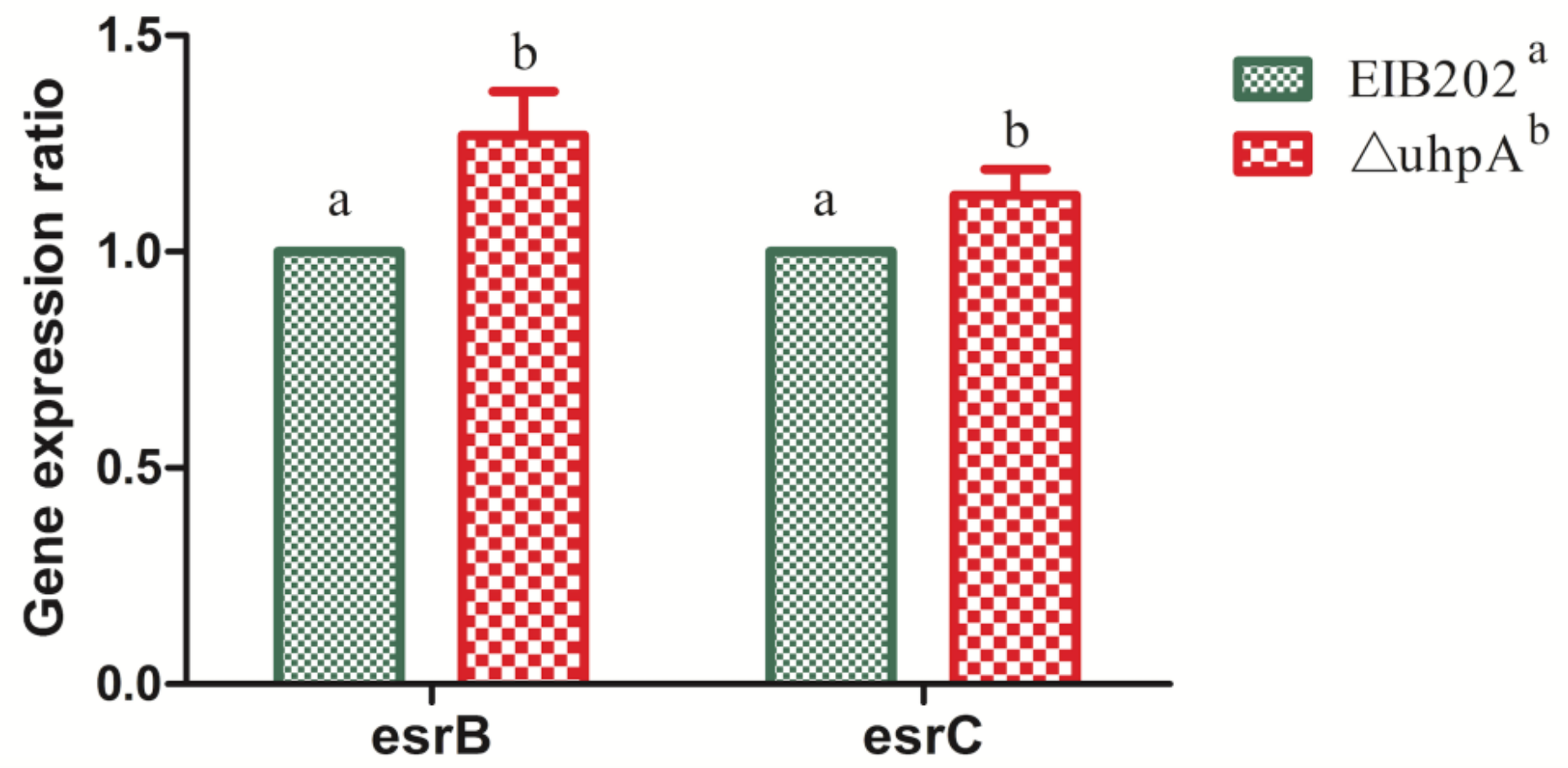

Figure 4

The difference of transcript levels of the key T3SS related genes in E. piscicida EIB202 and mutant strain $\triangle$ uhpA. Alphabet a and b: Values indicate significant difference at $P<0.05$.

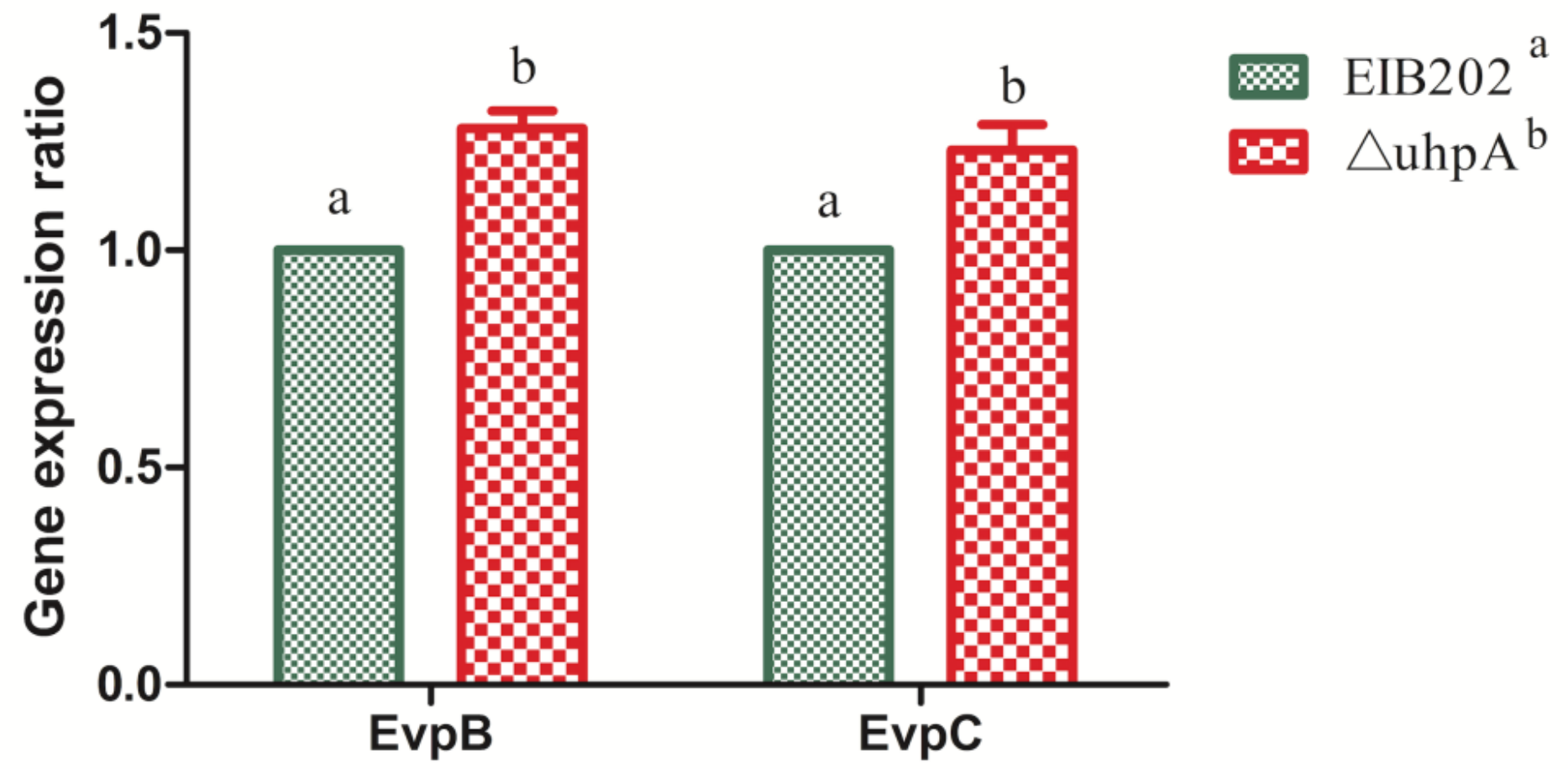


Figure 5

The difference of transcript levels of the key T6SS related genes in E. piscicida EIB202 and mutant strain $\triangle$ uhpA. Alphabet a and b: Values indicate significant difference at $\mathrm{P}<0.05$.

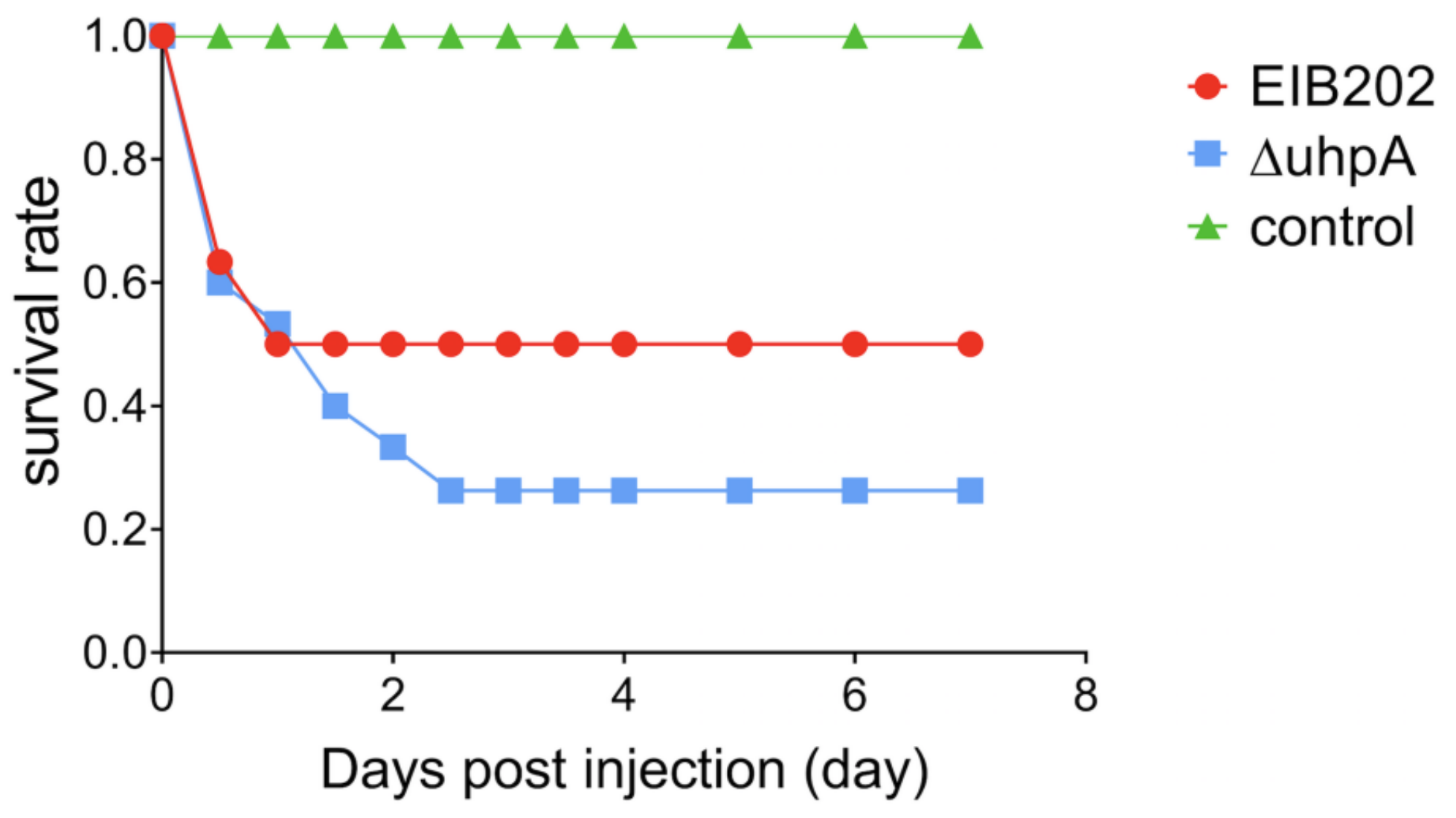

Figure 6

The difference of transcript levels of the key T6SS related genes in E. piscicida EIB202 and mutant strain $\triangle$ uhpA. Alphabet a and b: Values indicate significant difference at $\mathrm{P}<0.05$. 